\title{
A coupled modeling framework for predicting ecosystem carbon dynamics in boreal forests
}

Chao Huang ${ }^{1,2}$, Hong S. He ${ }^{3,4 *}$, Todd J. Hawbaker', Yu Liang ${ }^{1}$, Peng Gong ${ }^{6}$, Zhiwei Wu ${ }^{1}$, Zhiliang $\mathrm{Zhu}^{7}$

1. CAS Key Laboratory of Forest Ecology and Management, Institute of Applied Ecology, Chinese Academy of Sciences, Shenyang 110016, China

2. University of Chinese Academy of Sciences, Beijing 100049, China.

3. School of Natural Resources, University of Missouri, Columbia, MO, USA

4. School of Geographical Sciences, Northeast Normal University, Changchun 130024, China

5. U.S. Geological Survey, Geosciences and Environmental Change Science Center, Denver, CO, 80225 USA

6. Ministry of Education Key Laboratory for Earth System Modeling, Center for Earth System Science, Tsinghua University, Beijing 100084, China

7. U.S. Geological Survey, Reston, VA, 20192, USA

\begin{abstract}
Carbon stocks in boreal forests play an important role in global carbon balance but are sensitive to climate change and disturbances. Ecological models offer valuable insights into the effects of climate change and disturbances on boreal forests carbon stocks. However, the current pixel-based model coupling approaches are challenging to apply over large spatial extents because high computational loads and model parameterizations. Therefore, we developed a new framework for coupling a forest ecosystem and a landscape model to predict aboveground and soil organic carbon stocks at the ecoregion level. Results indicated that the new model-coupling framework has some advantages on computation efficiency and model validation. Our results showed that carbon stocks and its spatial distribution were significantly influenced by fire, harvest, and their interactions. Simulation results showed that boreal forest carbon stocks are vulnerable to loss because of future potential disturbances, complicating efforts to offset greenhouse gas emissions through forest
\end{abstract}


management.

Keywords: Model coupling, LINKAGES v2.2, LANDIS PRO, Fire, Harvest, Carbon stocks

\section{Software availability}

Name of software: LANDIS PRO

Developer: Prof. Hong S. He (heh@ missouri.edu)

Program language: $\mathrm{C}++$

Download http://landis.missouri.edu/

Name of software: LINKAGES v2.2

Developer: Dr. Stan D. Wullschleger (wullschlegsd@ornl.gov)

Program language: FORTRAN and $\mathrm{C}++$

Download http://daac.ornl.gov/cgi-bin/dsviewer.pl?ds_id=1166

\section{Introduction}

Boreal forests cover about one third of the global forest area, contain $32 \%$ of global forest carbon stocks (Pan et al., 2011), and play an important role in the global carbon balance (Bradshaw and Warkentin, 2015; Dixon et al., 1994). Field and model-based studies have shown that carbon stocks of boreal forests are highly sensitive to climate change and disturbances (Harden et al., 2000; Lutz et al., 2013; McGuire et al., 2009). Changes in boreal forest carbon stocks may significantly alter terrestrial ecosystem carbon balance and may lead to a positive feedback between climate change and carbon cycling (Goodale et al., 2002; Jones et al., 2005).

Therefore, future changes of boreal forest carbon stocks and reasons for those changes have emerged as an important research topic (Bond-Lamberty et al., 2007).

Modeling provides a unique approach for projecting forest ecosystem carbon dynamics. Forest ecosystem models have a tight coupling between physical and biological processes such as photosynthesis, growth, mortality, and decomposition while simulating aboveground carbon dynamics. They also include biogeochemical processes while simulating belowground carbon dynamics ( $\mathrm{Lu}$ and Cheng, 2009). Several forest ecosystem models were developed to predict boreal forest carbon stocks, including the Boreal Forests Carbon Dynamics Model (Nalder and Wein, 
2006), the Carbon Budget Model of the Canadian Forest Sector (Cameron et al., 2015; Chen et al., 2000; Kurz et al., 2009), the Individual based Spatially Explicit Simulation Model of Forest Ecosystem (Chertov et al., 2009), the Individual based forest ecosystem model LINKAGES (Post and Pastor, 1996) and the Terrestrial Ecosystem Model (Zhuang et al., 2002). These models offer valuable insights into the effects of climate change and disturbances on future potential changes in carbon dynamics. However, they typically have no, or simple treatment of forest landscape processes (typically disturbances and management) as well as stand-scale processes such as establishment and competition. Forest ecosystem carbon stocks are the result of interactions between forest growth, climate, soil, and forest landscape processes (Chen and Shrestha, 2012; McGuire et al., 2009; Smith et al., 2000; Ter-Mikaelian et al., 2013). These interactive processes control forest net primary production and litter decomposition, which in turn affect carbon stocks (Bonan and Cleve, 1992; Pregitzer and Euskirchen, 2004). For instance, fire and harvest may release carbon directly into the atmosphere and transfer a large amount of carbon from live biomass into detritus, soils, or forest products (Jandl et al., 2007; Kashian et al., 2006; Nave et al., 2010). Besides, repeated disturbances, such as fires and harvest may result in a large proportion of forest in young age classes which contain less carbon than mature stands (Pregitzer and Euskirchen, 2004; Yang et al., 2011). Moreover, timber harvesting after fire reduces the forest canopy and net primary production, which affects litter decomposition, carbon sequestration capacity, and nutrient cycling (Brais et al., 2000; Marañón-Jiménez and Castro, 2012; Serrano-Ortiz et al., 2011). Therefore, without considering these interactive processes, predictions made by forest ecosystem models may have high uncertainties.

Forest landscape models are designed to simulate landscape-level processes of seed dispersal, disturbance, management and their interactive effects on forest composition and biomass (He et al., 2005; Scheller and Mladenoff, 2005). Forest landscape models provide insight into the relationship between disturbances and aboveground biomass at landscape levels. However, most forest landscape models do not simulate forest ecosystem processes (especially biogeochemical processes) and 
thus, are limited in their ability to predict belowground carbon dynamics. Thus, coupling forest landscape and forest ecosystem models may provide viable alternatives to this problem. Loudermilk et al. (2013) and Lucash et al. (2014) coupled a forest landscape model (LANDIS-II) with an ecosystem process model (CENTURY) to integrate forest stand dynamics with belowground carbon and nitrogen processes. The coupled modeling approaches offer advantages over either model alone, such as disturbance-caused changes in species composition can affect soil nutrient dynamics (Scheller et al., 2011). However, the current model coupling approach creates challenges because of the overwhelming computational resources needed to process large landscapes. This is because the current framework of model coupling is at the pixel level and having to processes millions of pixels makes the simulation intractable (Fig. 1). In addition, most parameters related to biogeochemical cycling (soil water and nutrients) do not often exist at the pixel level, rendering model parameterization and validation difficult.

Most soil and hydrological data are usually available and follow natural boundaries such as landform, landtype, or ecoregion. Therefore, coupling forest ecosystem and landscape models at landform or ecoregion levels may not only improve parameter realism but also improve simulation efficiency since one landform or ecoregion unit may contain numerous pixels. In this paper, we propose a framework of coupling different ecological models at the ecoregion level, which may provide an alternative to current pixel based modeling framework. Thus, the objectives of this study were to (1) develop a new framework for coupling forest ecosystem and landscape models to predict aboveground and soil organic carbon at the ecoregion level; (2) validate this framework under the succession, fire, harvest and fire-harvest scenarios; and (3) apply this framework to quantify the effects of fire, harvest and their interactions on boreal forests carbon stocks.

\section{Materials and methods}

\subsection{Study area}

Our study area is located in the north side of the Great Xing 'an mountains of 
Northeastern China $\left(51^{\circ} 14^{\prime}-52^{\circ} 25^{\prime} \mathrm{N}, 122^{\circ} 39^{\prime}-124^{\circ} 21^{\prime} \mathrm{E}\right)$, about one million ha. This area covers an elevation gradient, ranging from $406 \mathrm{~m}$ in the northeast to 1,515 $\mathrm{m}$ in the southwest (Fig. 2). The climate is cold-temperate continental, with long and cold winters (mean January temperature $-32.3^{\circ} \mathrm{C}$ ) and short humid summers (mean July temperature $21.2^{\circ} \mathrm{C}$ ). The mean annual precipitation is $497 \mathrm{~mm}$, more than $68 \%$ of which occurs in summer.

This area is dominated by cool temperate coniferous forests, which belong to the southern extension of the Siberian light coniferous forest. Larch (Larix gmelinii (Rupr.) Kuzen) and white birch (Betula platyphylla Suk.) are dominant species in this region which cover over $90 \%$ of the forested area. Korean spruce (Picea koriensis Nakai), Scots pine (Pinus sylvestris var. mongolica Litvinov), aspen (Populus davidiana Dole), poplar (Populus suaveolens Fisch.), and willow (Chosenia arbutifolia (Pall.) A. Skv) cover less than $10 \%$ of forest in this area. Dwarf pine (Pinus pumila (Pall.) Regel) is mainly distributed on the ridges at elevations over $800 \mathrm{~m}$.

In this region, the main soil types are Umbri-geliccambosols, Alluvicprimosols and histi-orthicgleyosols (Cooperative Research Group on Chinese Soil Taxonomy, 2001). Umbri-geliccambosols make up about $95 \%$ of the forest area in this region, which contain a high level of humus, nitrogen and rock. The surface organic horizon $(0-10 \mathrm{~cm})$ is characterized by a litter layer or humus layer that contains more organic matter than mineral soil layer. The thickness of mineral soil layer ranges from 25 to $65 \mathrm{~cm}$.

Fire is the major disturbance in the Great Xing' an Mountains. According to historic wildfire records from the Chinese Forestry Science Data Centre (http://www.cfsdc.org/), human and lightning-caused fire burning 352,889 ha forests from 1967 to 2005 in this region. Since the 1960s, forest composition and structure have been dramatically changed by timber harvesting in this region. The cool temperate coniferous forests usually regenerate to white birch forests after stand-replacing fire or harvest. In this area, the average age of forests is 60 years, but age varies depending on disturbance history. 


\subsection{LINKAGES ecosystem model and parameterization}

LINKAGES v2.2 is a modified version of the forest ecosystem model developed originally by Pastor and Post (Post and Pastor, 2013). Specifically, LINKAGES v2.2 was modified to reflect results from the Throughfall Displacement Experiment (Wullschleger et al., 2003). The LINKAGES v2.2 model simulates changes in stand structure and the soil nutrient cycle based on allometric relationships with diameter to determine height, leaf area, and biomass. Regeneration is simulated for each species based on light, growing degree days, soil moisture multipliers, and a random number (Pastor and Post, 1986). Growth is simulated as maximum growth reduced by light, growing degree days, soil nitrogen, and soil moisture limiting factors. Mortality is simulated by annual tree growth of $\leq 10 \%$ of maximum possible diameter growth and by a background rate of the inverse of maximum typical longevity. LINKAGES v2.2 also simulates hydrological dynamics accounting for soil water holding, plant water use and evapotranspiration. Finally, LINKAGES v2.2 simulates soil carbon and nitrogen cycling by combining tree physiological processes, demographic processes, microbial processes and hydrologic processes (Ranatunga et al., 2008).

The climate parameters of LINKAGES v2.2 were derived from a 1975-2000 year China National Meteorological Monitoring Dataset (http://data.cma.gov.cn). These parameters include daily maximum and minimum temperature, daily precipitation amount, daily mean incoming solar radiation, and daily mean wind speed. These climate parameters were used to calculate the actual and potential evaporations according to Penman and Thornthwaite-Mather's method (Thornthwaite and Mather, 1957). The thickness of soil layers, soil texture, soil organic matter and soil nitrogen content were obtained from the China Soil Database (http://www.soil.csdb.cn). The soil field capacity and wilting point were estimated through soil texture. Species parameters and the length of growing season were obtained from previous studies ( $\mathrm{He}$ et al., 2005; Liang et al., 2011). Stand parameters (number of trees, age and DBH) were derived from simulation results of LANDIS PRO. 


\subsection{LANDIS PRO landscape model and parameterization}

LANDIS PRO is a spatially explicit forest landscape model that is designed to simulate forest succession, seed dispersal, natural disturbances and silvicultural treatments at various spatial and temporal scales (Wang et al., 2014). In this study, we used LANDIS PRO to predict aboveground carbon under scenarios including succession, fire, harvest and their interactions. The LANDIS PRO model estimates aboveground carbon from DBH and stand density by species using allometric equations. The input parameters of LANDIS PRO are completely compatible with forest inventory data, which makes it convenient to calibrate and validate model performance against field survey data (Wang et al., 2013).

In LANDIS PRO, the dynamics of forest succession is simplified and simulated as birth, growth, and death processes acting on individual species (Wang et al., 2014). At each pixel, the process of succession is controlled by species life history attributes and growing space occupied (GSO). LANDIS PRO tracks density (number of trees) and species age cohorts at each pixel. Such information is essential in simulating age increment and age-related mortality. With the age-cohort information, DBH of a given age cohort of each species can be derived from empirical log-normal relationships between species age and size. This design makes it possible to use mean DBH and density to calculate Reineke's stand density index (SDI). Growing space occupied (GSO) is a percentage of a pixel physically occupied by trees, which is estimated by stand density index (SDI) and pixel size. Four degrees of stand competition status (stand initiation, stem exclusion, understory re-initiation, and old-growth) are determined by GSO.

The fire module of LANDIS PRO includes fire occurrence, spread and effects submodules. The fire occurrence submodule uses a statistical distribution of fire frequency to simulate the temporal patterns of fire regimes (Yang et al., 2004). The fire spread submodule has two algorithms to calculate fire spread. The first one is a percolation algorithm which simulates fire spread from a burning pixel to another pixel in the cardinal directions. The second algorithm simulates fire spread behavior 
by analyzing fuel configuration, topography, wind speed and direction (Yang et al., 2008). In the fire effects simulation submodule, fire intensity is determined by the quantity and quality of fuel. The effects of fire on individual trees and age cohorts depend on species fire tolerance class and age susceptibility.

Timber harvesting is conducted using management area and stand maps in the harvest module (Gustafson et al., 2000). The harvest module of LANDIS PRO has three ranking algorithms to select stands for logging (Fraser et al., 2013). The first is a basal area ranking algorithm where eligible stands are harvested to meet timber harvesting goals. The second ranking algorithm is controlled by the stand stocking level. This algorithm applies the Gingrich stand density stocking equation to calculate forest harvesting volume (Johnson et al., 2009). The third ranking algorithm is a group selection method, which is designed to create canopy openings in a stand based on forest management objective.

Eight tree species were simulated in LANDIS PRO and LINKAGES models, which accounted for approximately $97 \%$ of stand volume in this area. The species life history attributes were derived from previous studies (Li et al., 2013; Luo et al., 2015). The species composition map consisted of the number of trees and age cohorts that were generated from a forest type map and China National forest inventory data. In LANDIS PRO model, the seedling establishment was controlled by species establishment probability (SEP) that was obtained from previous studies (Li et al., 2013; Luo et al., 2014).

The main parameters of fire module in LANDIS PRO included mean fire return intervals, mean fire size, and fire ignition probability. These parameters were obtained from a historical fire database from 1967 to 2005 in our study area. According to the historical wildfire record from China Forestry Science Data Central (http://www.cfsdc.org), the mean return interval is 238 year, mean fire size is 1,880 ha, and fire ignition probability is 0.0040 in our study area (Li et al., 2013; Liu et al., 2012). We assumed that the fire parameters remained unchanged over the whole simulation period.

The main parameters in harvest module included the type of management area, 
harvest method, harvest rotation, the target size of harvest, and the type of regeneration. Our study area was divided into three management areas (forbidden harvest areas, restricted harvest areas, and commercial harvest areas). According to the forest management plan, larch, birch and aspen were permitted for harvest. Scotch pine, Korean spruce, poplar, willow, and dwarf pines were not permitted for harvest. The main harvest method was clearcut. Each management area was further divided into stands, the basic unit for harvesting operations, with an average size of 20 ha. The minimum stand age to harvest was 40 years according to the forest management plan in this region (Luo et al., 2015). In this study, we assumed that the forest management plan and timber harvest methods remained unchanged.

\subsection{The framework of model coupling}

LANDIS PRO model can be used to simulate the dynamics of forest landscape patterns and aboveground carbon stocks over large spatial and temporal scales. However, LANDIS PRO cannot simulate soil organic carbon and nitrogen cycling because it lacks a biogeochemical module. The LINKAGES model includes tree birth, growth, mortality, decomposition, mineralization, and soil moisture subroutines, and allows water and soil carbon cycles to interact with species composition. The LINKAGES model simulation results compare favorably to field data from different areas worldwide, but it cannot simulate disturbance and forest management (Pastor and Post, 1986). Therefore, we coupled LANDIS PRO with LINKAGES model to predict aboveground carbon and soil organic carbon under different disturbance scenarios.

We coupled the LANDIS PRO and LINKAGES models at the ecoregion level. Our study area was classified into 26 ecoregions based on topography and soil conditions (Fig. S1). Each ecoregion has homogenous soil type, soil nutrient status and climate conditions. We simulated the dynamics of stand structure and aboveground carbon under the succession, fire, harvest and fire-harvest scenarios using LANDIS PRO at the ecoregion level. The stand parameters, including the number of trees, age and DBH from LANDIS PRO, were input into LINKAGES to 
predict soil organic carbon, available nitrogen, and water for each ecoregion (Fig. 3).

\subsection{Simulation scenarios and data analysis}

We designed four simulation scenarios to explore the effects of fire, harvest and their interactions on boreal forests carbon stocks: (1) succession scenario (the effects of fire and harvest were not simulated); (2) fire scenario (simulating succession and fire); (3) harvest scenario (simulating succession and harvest), and (4) fire-harvest scenario (simulating succession, fire, and harvest). Each scenario was simulated from 2000 to 2300 at 10 year time-steps with five replicates to assess the model uncertainty (Liang et al., 2014). We added a reference simulation that further divided the 26 original ecoregions into 45 to assess the sensitivity of simulation results to ecoregion size. The study area was divided into 45 ecoregions based on topography, forest type and soil conditions (Fig. S2).

We compared simulation results from the succession, fire, harvest, and fire-harvest scenarios to evaluate the effects of fire, harvest and their interactions on boreal forests carbon stocks. To assess the influence of fire, harvest and their interactions on forest carbon stocks at different time periods, simulation results were analyzed at the short-term (0-50 year), medium-term (50-100 year), and long-term (100-300 year). The Least Significant Difference (LSD) method was used for post-hoc analyses among three ecoregions at the short-term, medium-term and long-term time periods (Luo et al., 2014). We used analysis of variance (ANOVA) to test for differences among simulation results derived from 26 and 45 ecoregions under the four simulation scenarios. The $\mathrm{R}$ statistical software was used in the data analyses ( $R$ Core Team, 2015).

\subsection{Model validation}

We used forest inventory data to estimate aboveground carbon based on previous studies (Hu et al., 2012; Wang et al., 2001). Then we compared observed aboveground carbon with our simulated results to validate the LANDIS PRO model. A total of 112 independent forest inventory plots were selected to evaluate the simulation results for the year 2000. In addition, we randomly selected 136 pixels from the simulated 
aboveground carbon map for year 2010 to compare with the forest inventory plots from 2010.

We used soil organic carbon data to verify simulated results of the LINKAGES model. These data included: (1) data from 34 soil plots that were sampled in August 2012 in our study area; (2) 179 soil plots from four sites in Northeast China (Genhe, our study area, Yichun and Changbai Mountains) (Wei et al., 2013); (3) simulation results from a forest ecosystem model (TRIPLEX) that was used to investigate the impacts of climate change and increasing atmospheric $\mathrm{CO}_{2}$ on forest carbon budgets in Northeast China (Peng et al., 2009).

\section{Results}

\subsection{Model validation}

The initial and simulated aboveground carbon stocks were similar to observed values derived from the forest inventory plots sampled in 2000 and 2010.

Comparison between initialized aboveground carbon and observed data at year 2000 showed that the initialized aboveground carbon was close to the observed values from the forest inventory data (Fig.4a); correlations between the year 2000 datasets was 0.83. The simulated aboveground carbon was close to the observed forest inventory data at year 2010 (Fig.4b); correlations were high with $\mathrm{R}^{2}=0.82$.

To validate the soil organic carbon stocks simulated by the LINKAGES model, we compared our results with field data and simulation results from the TRIPLEX model. The results showed that the values from LINKAGES were within the range of the TRIPLEX values reported by Peng (2009) and the soil plots measured by Wei (2013), and close to the average value of the 34 soil plots we measured (Fig. 5).

\subsection{The sensitivity of results from different ecoregion size}

The results of ANOVA showed that there was no significant difference in the aboveground and soil organic carbon at the two ecoregion size (Fig.6, $\mathrm{P}<0.05$ ). At the landscape level, aboveground carbon was $37.9 \pm 3.7,35.4 \pm 3.9,33.8 \pm 3.3$ and $30.8 \pm$ $2.1 \mathrm{t} /$ ha for the four simulation scenarios in the 26 ecoregions, respectively. Meanwhile, 
aboveground carbon was $38.6 \pm 3.9,36.2 \pm 3.4,34.1 \pm 3.3$ and $31.2 \pm 2.1 \mathrm{t} / \mathrm{ha}$ under the four simulation scenarios in the 45 ecoregions, respectively. The average of soil organic carbon was $106.2 \pm 5.0$ and $109.4 \pm 4.8$ tha for the four simulation scenarios in the 26 and 45 ecoregions, respectively.

\subsection{The effects of fire and harvest on aboveground carbon}

Aboveground carbon was substantially lower under the fire, harvest, and fire-harvest scenarios than the succession scenario (Fig. 7). Our results showed that fire, harvest and their interactions resulted in 0.6-9.4 t/ha less aboveground carbon on average over the 300-year simulations. The largest reduction in aboveground carbon occurred in the harvest and fire-harvest scenarios, accounting for $11 \%$ and $23 \%$ of the average value of simulation results, respectively. In the fire-harvest scenario, aboveground carbon was lower than that in the individual fire and harvest scenarios. Aboveground carbon under the harvest scenario was lower than the fire scenario during the first 210 years, but was higher than the fire scenario after that.

Aboveground carbon responses to fire, harvest and their interactions differed among eight species. In the four simulation scenarios, aboveground carbon of larch, scots pine and Korean spruce increased with simulation time, whereas aboveground carbon of white birch, aspen and dwarf pine decreased (Fig.8). Among four simulation scenarios, aboveground carbon of poplar and willow firstly increases and then decreases with simulation time. There was a significant decrease in aboveground carbon of white birch under the succession scenario after 150 years. In contrast, aboveground carbon of scots pine and Korean spruce increased markedly after 150 years under all scenarios. Under the fire, harvest and fire-harvest scenarios, aboveground carbon of white birch was greater than that under the succession scenario. Fire, harvest and their interaction scenarios reduced the aboveground carbon of larch and white birch the most.

There was a significant difference in aboveground carbon between the fire-harvest and succession scenarios $(\mathrm{P}<0.05$, Fig. 9). Over the short-term $(0-50$ year $)$, aboveground carbon in the harvest scenario was $9 \%$ and $13 \%$ lower than that in the 
fire and succession scenarios, respectively. Aboveground carbon in the fire scenario differed significantly from the succession scenario over the medium and long term, which decreased between $5 \%$ and $8 \%$ across the medium to long-term range. Aboveground carbon under the harvest scenario also differ significantly from the succession scenario over medium and long term $(\mathrm{P}<0.05)$.

\subsection{The effects of fire and harvest on soil organic carbon}

Soil organic carbon increased over time under all four scenarios (Fig. 10). Similar to aboveground carbon, there was less soil organic carbon under the fire, harvest and fire-harvest scenarios compared to the succession scenario. The fire-harvest scenario reduced soil organic carbon the most. The fire scenario had the lowest reduction in soil organic carbon over 300 years.

There were no significant differences in soil organic carbon between the four scenarios for the short term (Fig. 11). In the medium term, soil organic carbon was significantly greater in the succession scenario than that in the fire, harvest, and fire-harvest scenarios. The results of the least significant difference tests indicated that soil organic carbon differed significantly among all four scenarios over the long term $(\mathrm{P}<0.05)$.

\subsection{The spatial patterns of aboveground and soil organic carbon over time}

In our study area, the spatial distribution of boreal forest carbon stocks varied substantially among three time periods and ecoregions (Fig. 12 and Fig. 13). The spatial patterns of aboveground carbon and soil organic carbon were visibly different among the scenarios after 50,150 and 300 years (Fig. 10). In the terrace ecoregion (ecoregion-1), aboveground carbon varied little among the four simulation scenarios at short-term (Fig. 13). In the north-slope ecoregion (ecoregion 17), aboveground carbon significantly varied among the four simulation scenarios in the medium and long term. Aboveground carbon under the fire-harvest scenario was lower than that in the succession scenario among three ecoregions in the medium and long term. In the terrace, south-slope and north-slope ecoregions, soil organic carbon under the 
fire-harvest scenario were significant lower than that in succession scenario in long term (Fig. 13, $\mathrm{P}<0.05$ ). For these three ecoregions, the largest reduction in soil organic carbon occurred in the fire-harvest scenario over the long term.

\section{Discussion}

We coupled a forest ecosystem model (LINKAGES) and a forest landscape model (LANDIS PRO) at the ecoregion level to predict boreal forests carbon stocks. The results showed that this coupled modeling framework is effective in predicting aboveground and soil organic carbon stocks. There are a number of advantages in this model coupling framework compared with the current pixel level model coupling framework. First, simulation results of our model coupling framework are verifiable against field inventory data, published and other model results. These may include stand maps, soil surveys, and hydrological data available as polygons, which usually follow natural boundaries. It is possible to apply multi-source data (field survey data, the published data, and other model results) to validate model results at the ecoregion scale. Second, the computation speed of our model coupling framework is about 5 times faster (20 days vs. 4 days) than the pixel-level model coupling framework. This is because the pixel-level model coupling framework needs to simulate millions of pixels at each time-step. In contrast, our model coupling framework simulates a much smaller number of ecoregions for the whole simulation period.

Our simulated results were consistent with field data and other model results at the landscape level. For example, simulated aboveground carbon under the four simulation scenarios (21-41 t/ha) is close to values from a study in the Great Xing 'an Mountains (15-45 t/ha) (Qi Yujiao, 2015). Soil organic carbon (100-119 t/ha) simulated by the LINKAGES model is similar to results from an existing study (129 t/ha) in the Great Xing 'an Mountains (Li et al., 2004). However, our simulated soil organic carbon values were lower than those (498.8 t/ha) reported from boreal forests in Northeast China (Wang et al., 2002). This discrepancy in soil organic carbon may be due to different environment conditions at the soil sampling sites. Differences in climate, vegetation, topography and disturbance regimes contribute to the differences 
between soil carbon pools of the boreal forest (Goulden et al., 1998; Johnson et al., 2011). Therefore, it is necessary to take vegetation change and disturbance into consideration to improve the accuracy of estimation of forest carbon stocks. The results of ANOVA showed that simulation results were not sensitive to different ecoregion size in our model coupling framework (Fig.6). This is because most environmental variables such as downscaled climate and soil survey data do not exist at multiple scales. Thus, dividing ecoregions into smaller units do not alter the thematic information of these data, while aggregating ecoregions into larger spatial units may have some lumping effects that are not significant in our study.

Our results showed that the fire and harvest simulation scenarios resulted in $0.2 \%$ to $13.4 \%$ less aboveground carbon over 300 years compared to the succession scenario. This is because fire and harvest not only lead to the loss of aboveground carbon, but also alter species composition and age distribution, which subsequently affects forest carbon sequestration (Shanin et al., 2013). Our results are generally consistent with previous studies in Canada, south-central Siberia and China (Chertov et al., 2009; Gustafson, 2010; Luo et al., 2014). For example, Chertov et al. used the EFIMOD model to simulate the effects of fire and timber harvest on aboveground biomass. Their results showed that fire and harvest decreased aboveground biomass 22.5-33.7 t/ha between 1961 and 2100. Gustafson et al. (2010) found that timber harvest and insect outbreaks significantly reduced aboveground carbon in Siberian boreal forests. Meanwhile, our results suggested that fire and harvest have a significant influence on boreal forests soil organic carbon. Fire, harvest and their interaction scenarios had less soil organic carbon $(104.1 \pm 2.4 \mathrm{t} / \mathrm{ha})$ than the succession scenario (112.3 $\pm 5.9 \mathrm{t} / \mathrm{ha})$. These results are consistent with previous findings (Bhatti et al., 2002; O'Donnell et al., 2011; Poirier et al., 2014; Thornley and Cannell, 2004). For example, Bhatti et al (2002) used the Carbon Budget Model (CBM-CFS2) to analyze the effects of nutrients, disturbances and site conditions on carbon stocks along a boreal forest transect in central Canada. Their study found that in the boreal forest zone, carbon stocks were strongly influenced by the disturbance pattern and tightly constrained by $\mathrm{N}$ availability. Some field studies showed that disturbances 
have no significant effects on soil organic carbon and nitrogen stocks (Piirainen et al., 2015).However, most field studies, conducted over a few decades, do not provide enough information to understand the long-term impacts of disturbances on soil organic carbon (Clarke et al., 2015). The discrepancy between our simulation results and some field studies may be because these field studies did not include long-term changes in soil organic carbon after disturbances. Properly obtained long-term data from new field studies can provide more valuable information on the mechanisms responsible for observed contrasting effects of fire and harvest on soil organic carbon, which can then be used for parameterization and validation of existing models. Thus, there is an urgent need to understand how disturbances affect forest ecosystem carbon stocks and sequestration over long time periods.

Our simulation results showed that the pioneer species (white birch and aspen) established quickly on the open growing space after fire or harvest. The shade tolerant species (larch, Scotch pine and Korean spruce) then regenerated and coexisted with the pioneer species over the next 50-100 years. Under the succession scenario, the short-lived pioneer species (120-150 years) gradually died and gave way to longer-lived shade-tolerant species (250-300 years). Finally, forest succeeded to a climax community characteristic of these coniferous forests (Fig.8). This is consistent with a pervious study in the Great Xing' an Mountains (Xu, 1998). In the fire, harvest and fire-harvest scenarios, fire and harvest not only removed live trees, but also reset succession process in boreal forests of the Great Xing 'an Mountains. Over long term, repeated fire and harvest resulted in forests dominated by larch, Scotch pine and white birch. These results suggest that fire, harvest and their interactions may affect boreal forests carbon stocks by altering species composition and succession trajectory at landscape level.

Our results indicate that the spatial distribution of aboveground and soil organic carbon could change significantly over time under the four simulation scenarios (Fig. 12). We believe the divergent response of carbon stocks among ecoregions was due to the spatial interactions between fire, harvest, and seed dispersal, as has been found by previous studies (Scheller and Mladenoff, 2005). Our results showed that fire and 
harvest interactions markedly decrease soil organic carbon in the long term among three ecoregions (Fig. 13). This is because repeated fire and harvest result in a greater proportion of forest stands in young age classes, which contain less carbon than mature stands (Pregitzer and Euskirchen, 2004). Besides, our results suggested that there was no significant difference in aboveground carbon between succession, fire, harvest and fire-harvest scenario in ecoregion-1 with relatively low disturbance frequency and severity.

Our results showed that disturbances and their interactions reduced aboveground and soil organic carbon stocks. This implies that much of the carbon stored in vegetation and soil was released to the atmosphere or transferred from live biomass to forest products, which may have a negative influence on the regional carbon balance. In addition, disturbances cause high levels of tree mortality that may significantly reduce the ecosystem services forests provide. Therefore, forest management may need to consider the effects of disturbances on forest carbon dynamics, while at the same time, enhancing forest ecosystem productivity, increasing resilience to climate change, and reducing feedbacks that further enhance carbon loss.

In this study, we focused on assessing the effects of fire, harvest, and their interactions on stand age and species composition which have important effects on forest carbon stocks. The effects of fire and harvest on soil physical and chemical properties were not taken into consideration in the model coupling framework. However, fire and harvest have an appreciable impact on the physical and chemical properties of soil, such as soil bulk density, soil water holding capacity, $\mathrm{PH}$, and nitrogen content (Neary et al., 1999; Schmidt et al., 1996). The physical and chemical properties of soil may not only alter rates of soil organic matter decomposition and soil respiration, but also indirectly impact tree recruitment after disturbance (Johnstone and Chapin, 2006; Yanai et al., 2003). So, our study may underestimate the effects of fire and harvest on forest carbon dynamics. Moreover, the LANDIS PRO and the LINKAGES model coupling simulate the dynamics of forest carbon stocks without land-use change and global warming, which may impact the forest ecosystem carbon cycle. Incorporating these factors into model simulations may help improve 
our understanding of how disturbances affect long-term forest carbon dynamics.

\section{Conclusions}

In this study, we developed a model coupling framework to predict aboveground and soil organic carbon stocks in boreal forests at the ecoregion level. This method provides insight into boreal forest carbon stocks under the different disturbance scenarios. In summary, we concluded that (1) the new model coupling framework has a lot of advantages in model validation and computational efficiency compared with the current pixel-level model coupling framework; (2) the model coupling framework can be successfully implemented in boreal forests of Northeastern China; (3) simulation results are comparable to field survey data, published data, and other model results at the landscape level; (4) aboveground and soil organic carbon were significantly reduced by fire, harvest, and their interactions over 300 years; (5) fire, harvest and their interactions strongly influence the spatial distribution of boreal forest carbon stocks; (6) the accuracy of projections of boreal forests carbon stocks can be improved by considering the effects of fire, harvest and their interactions.

\section{Acknowledgments}

This works was supported by the USGS-MOST project and Chinese National Science Foundational Project (Nos. 41371199, 31570461 and 31570462). Any use of trade, product, or firm names is for descriptive purposes only and does not imply endorsement by the U.S. Government. The authors thank the workgroup from the HuZhong Forestry Bureau for field investigations. 


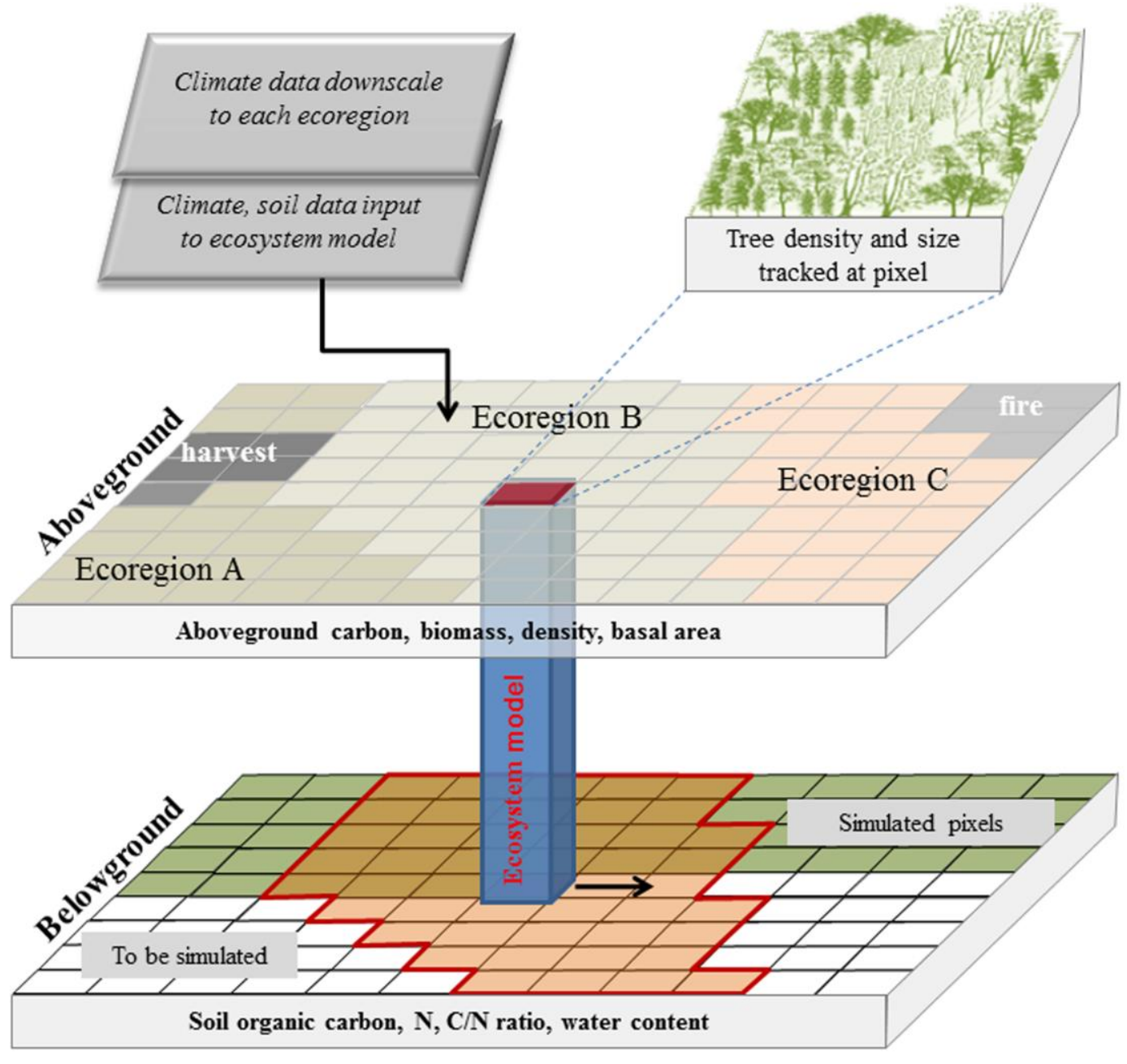




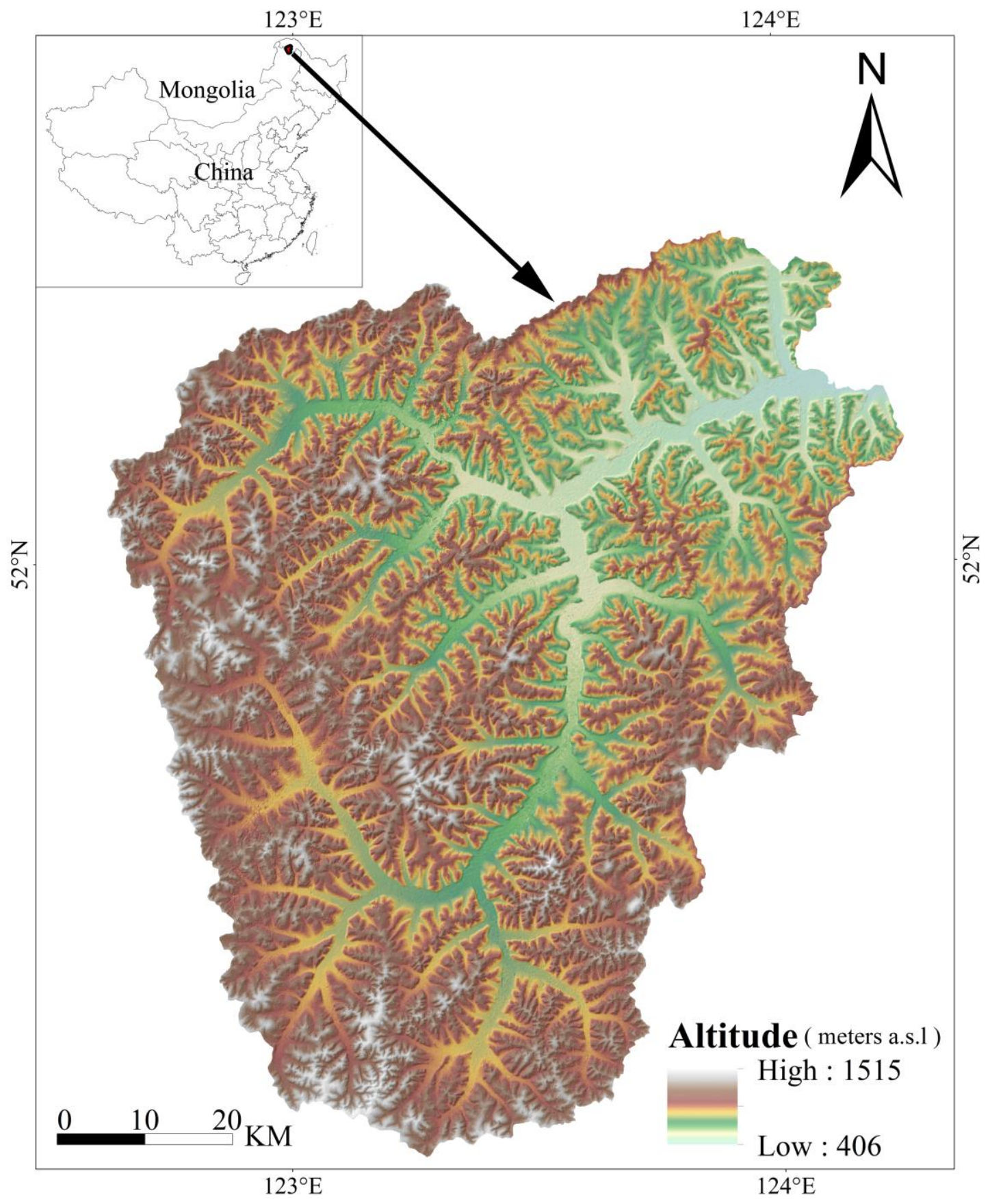




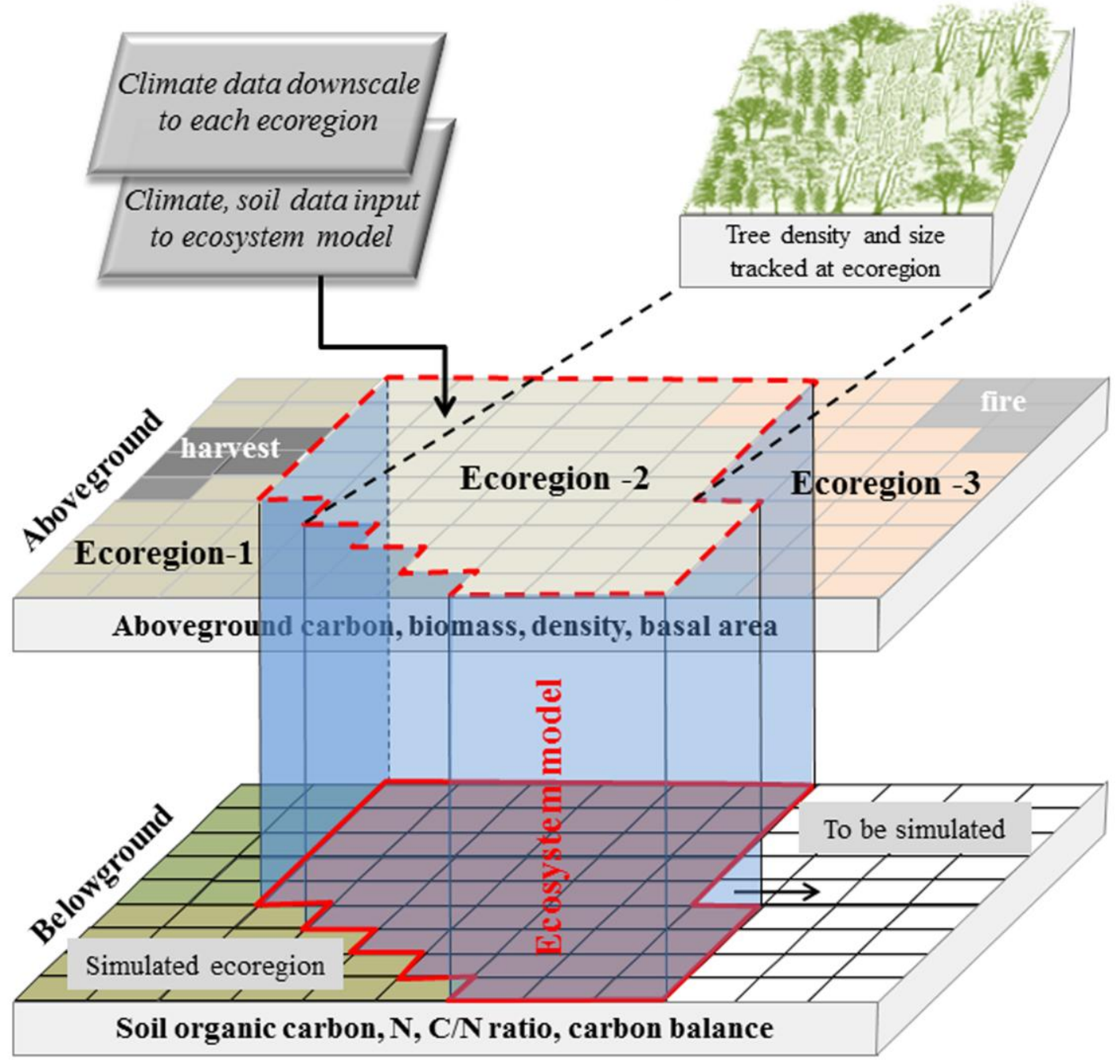



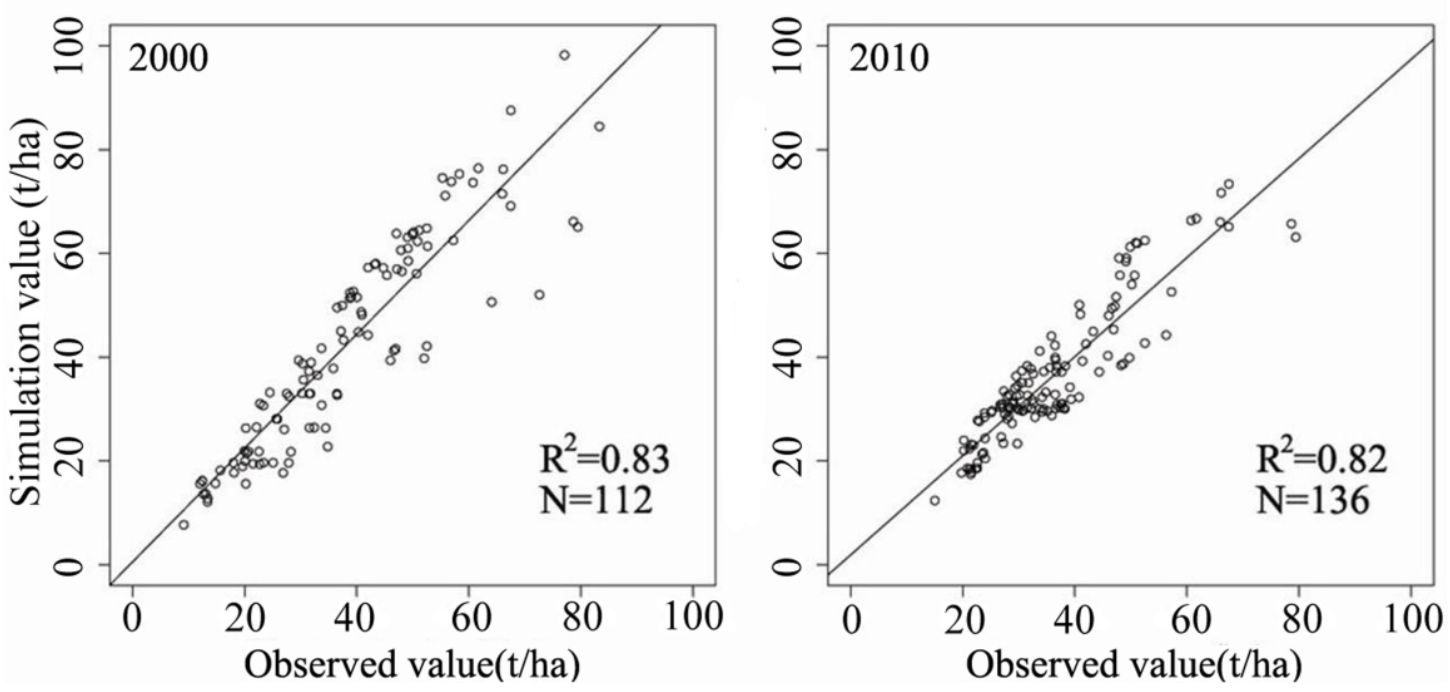


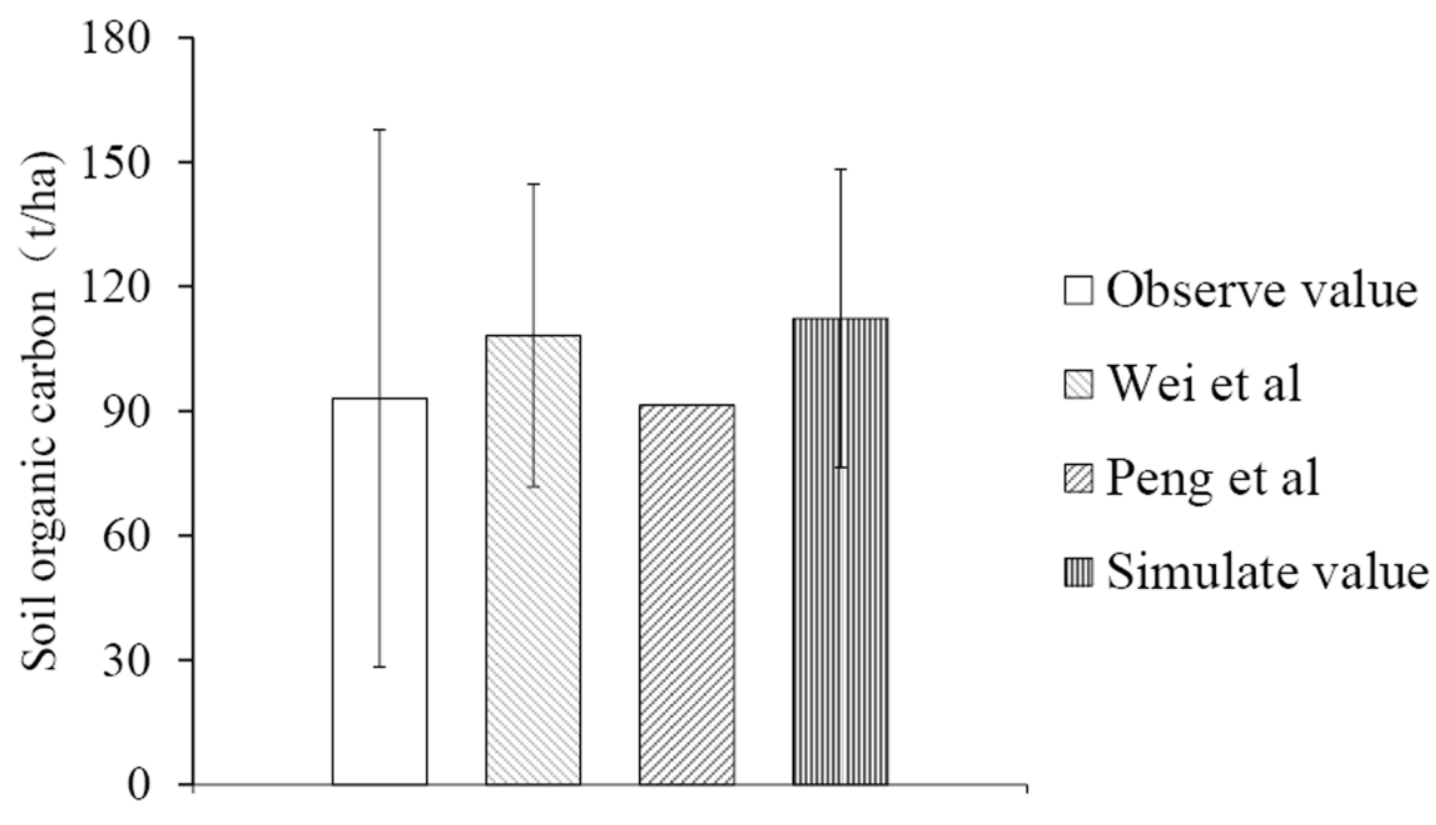




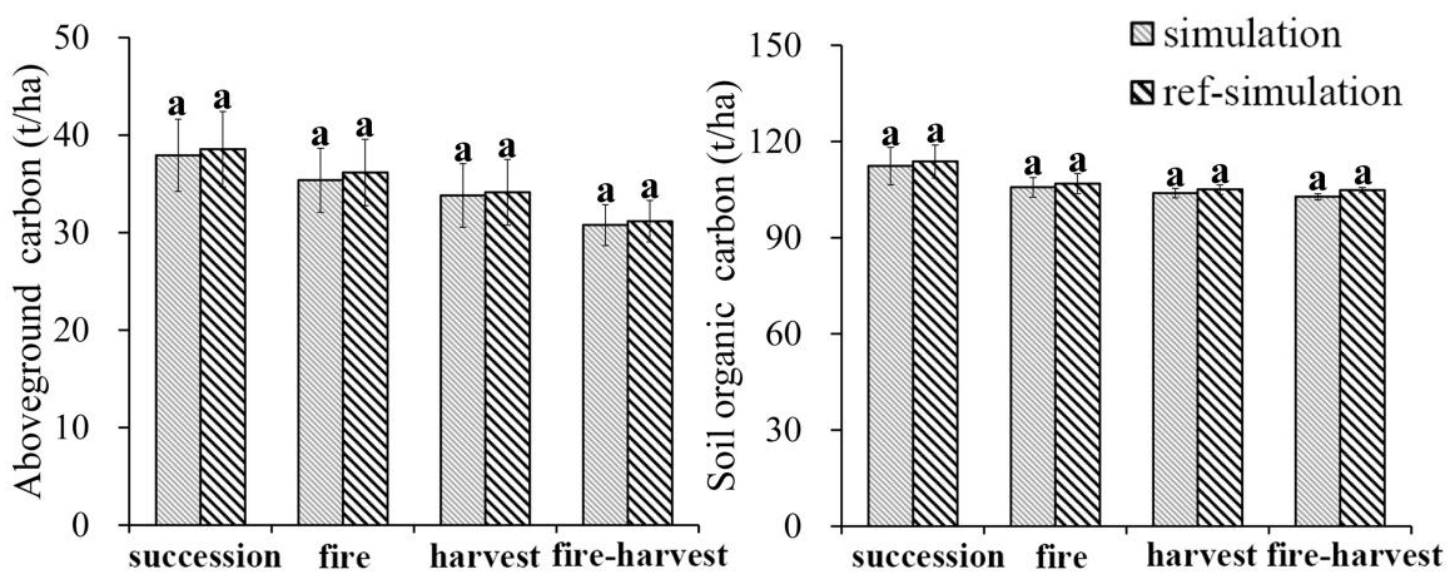




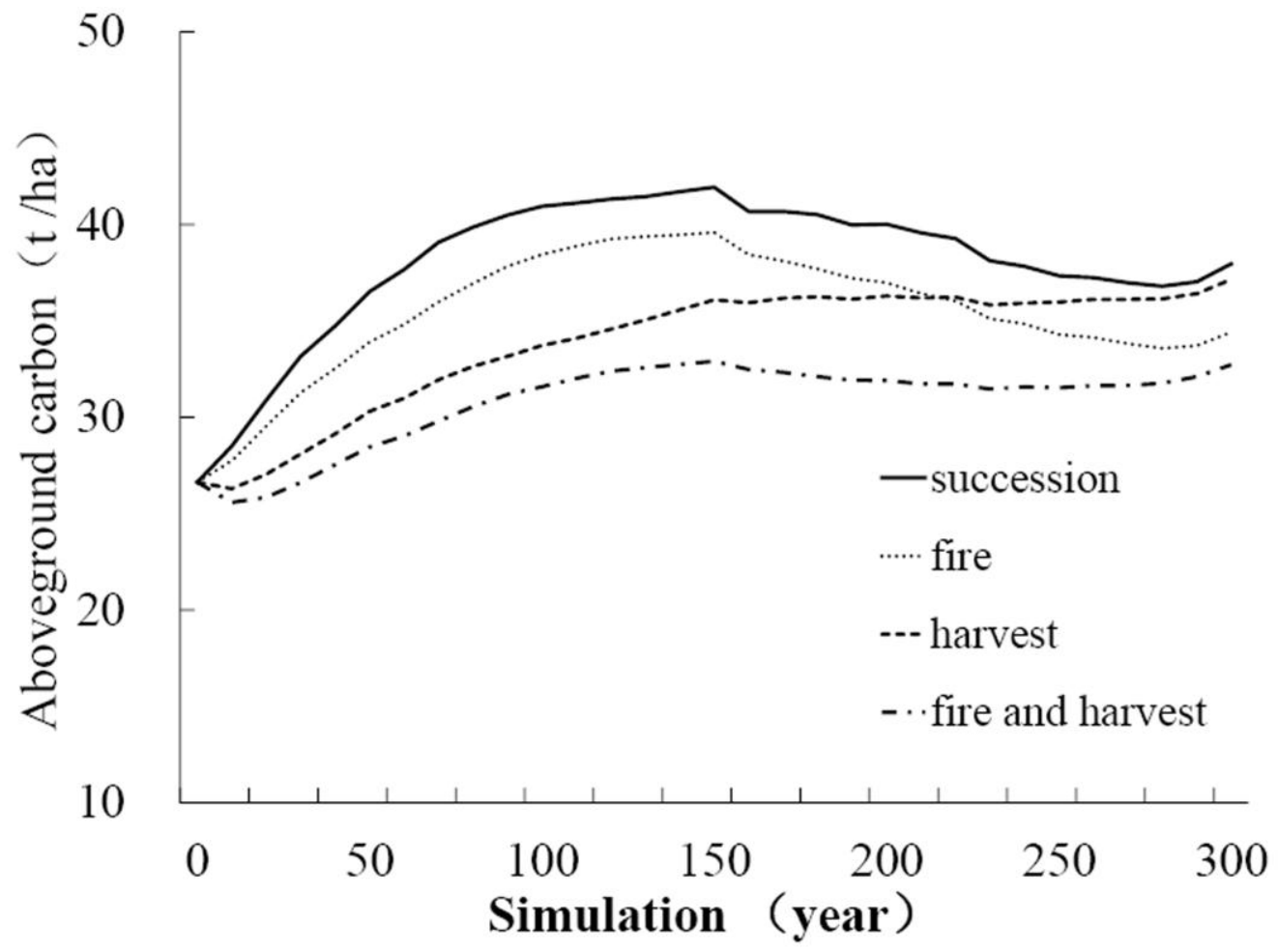




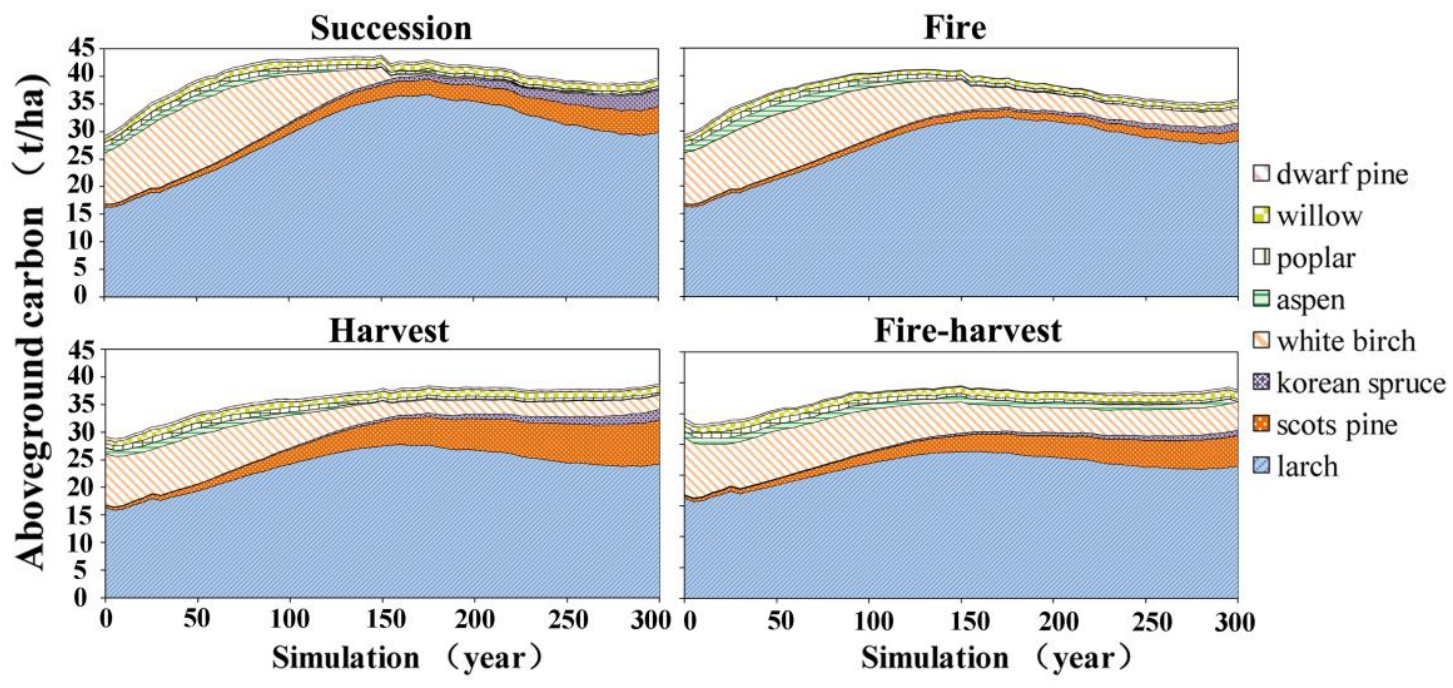



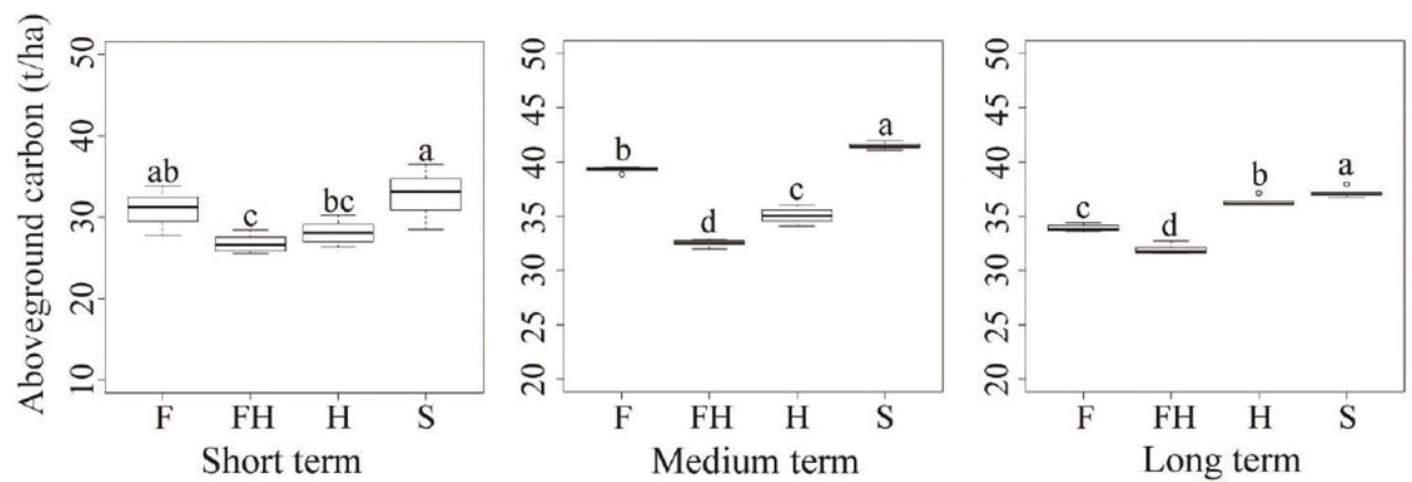


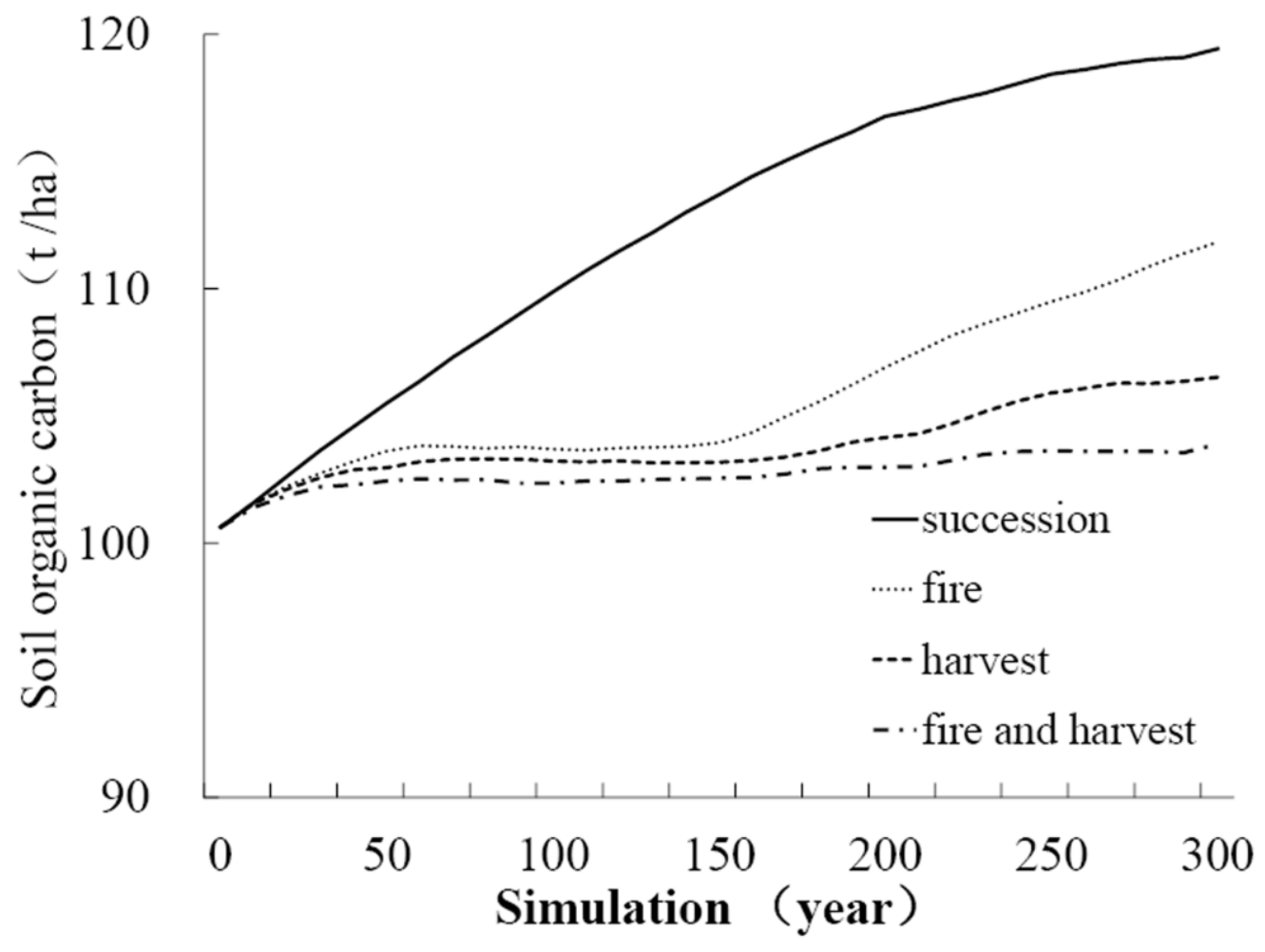



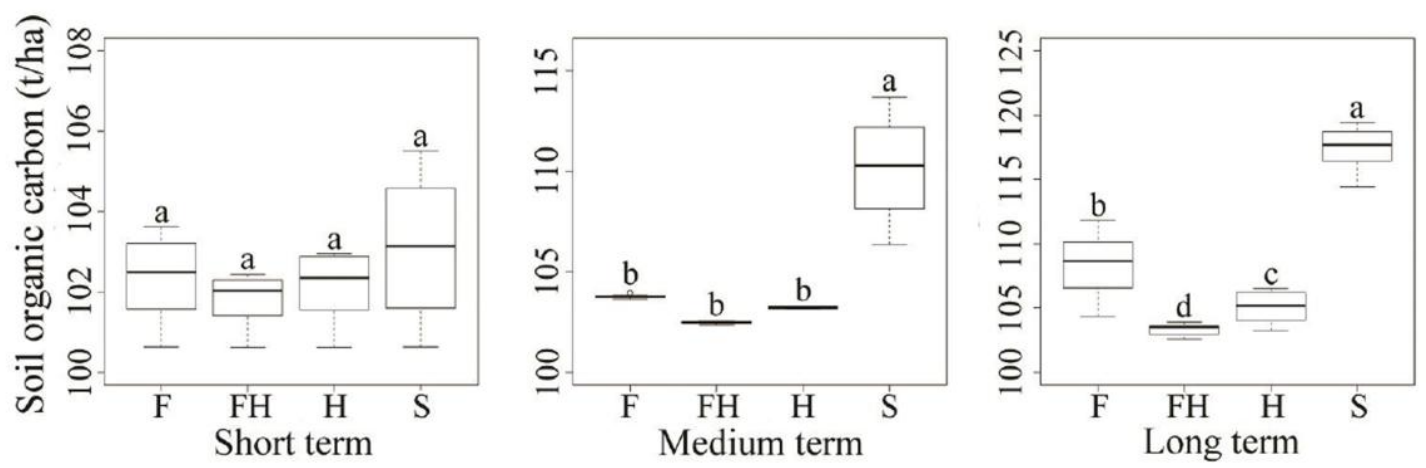


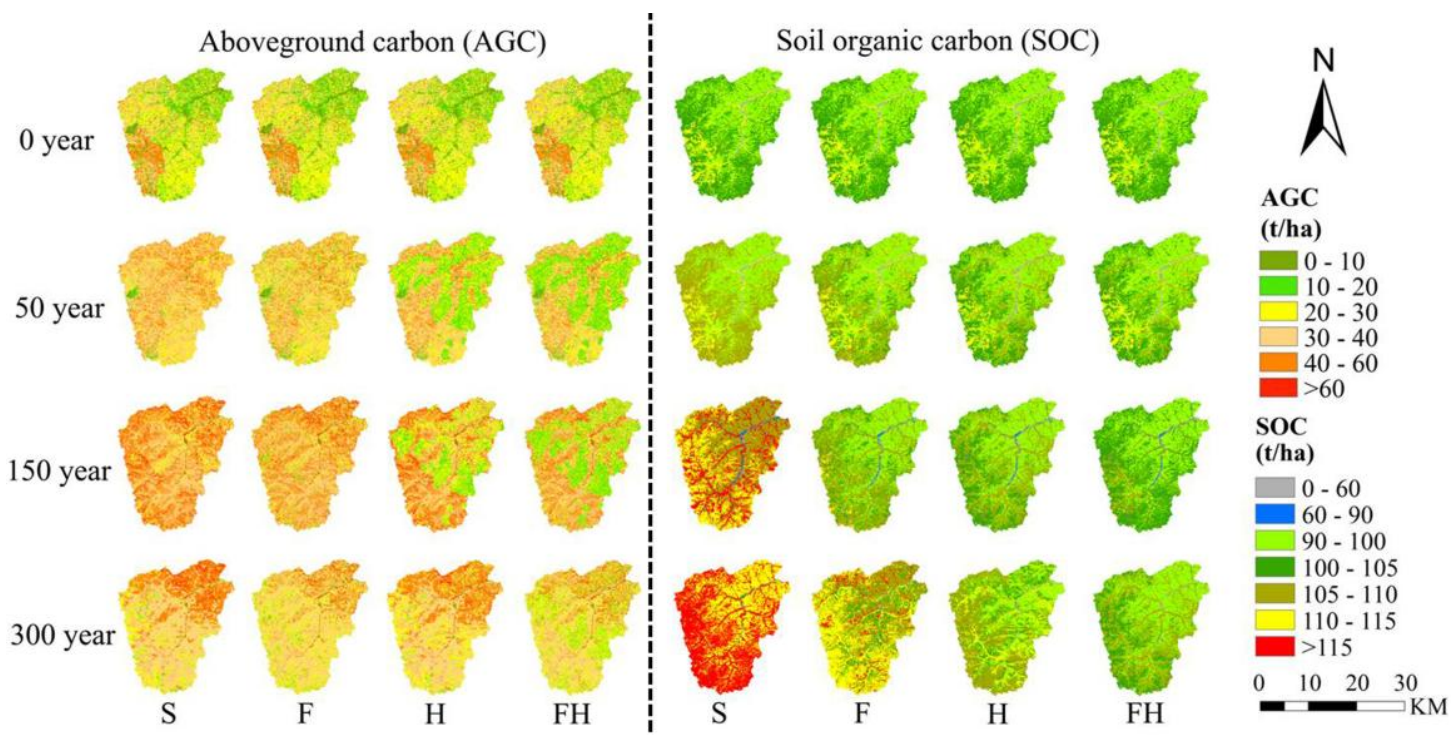




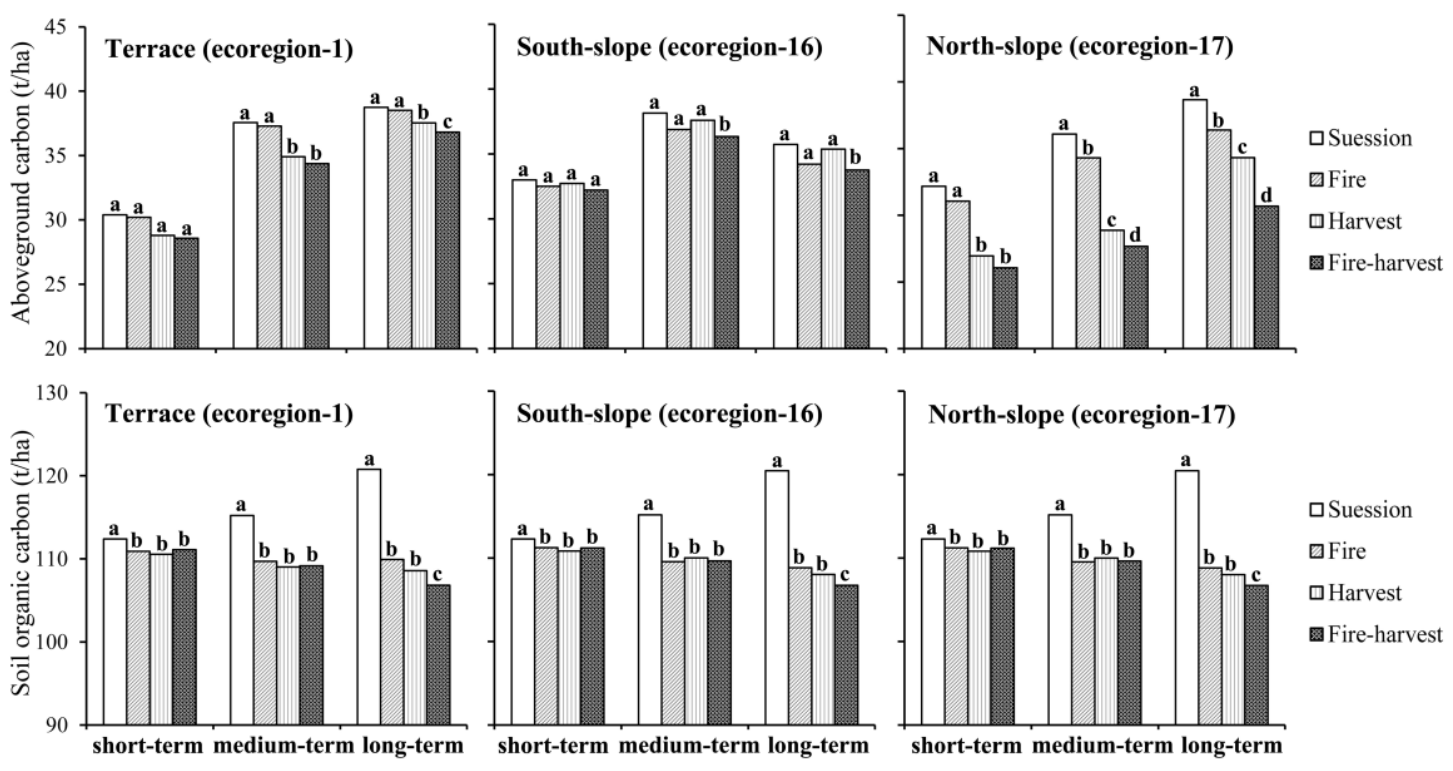

